\title{
Vigilia y vigilancia: Análisis de contenido del Registro Nacional de Acceso a Internet en Brasil
}

\section{Watch and surveillance: A content analysis of the National Registry of Internet Access in Brazil}

Fernando Nobre Cavalcante es doctorando en Estudios de los Medios en la Universidade Federal do Rio Grande do Norte y docente de la UNI7 (Brasil) (fernandocavalcante@gmail.com) (http://orcid.org/0000-00032803-52911

\begin{abstract}
Resumen
La legalización e institucionalización de la vigilancia de las informaciones digitales de los ciudadanos brasileños han sido promovidas por intereses de grupos políticos protestantes que argumentan ideas de fe y moralidad en proyectos de leyes que potencian nuevas formas de control de Internet. Desde 2015, diferentes proyectos de ley amenazan el derecho a la privacidad y a la libertad de expresión en las comunicaciones digitales, garantizados por la Ley No 12.965, del 23 de abril de 2014 (Marco Civil de Internet). La presente investigación analiza los puntos críticos de los proyectos de normativas de control de Internet promovidos por políticos evangélicos y justificados en argumentos morales de protección de niños y adolescentes. El presente artículo establece un diálogo teórico con los postulados de Manuel Castells en un intento por desmitificar los argumentos a favor del control y utiliza una investigación cualitativa por medio de un levantamiento y análisis de contenido de los proyectos de leyes que aún se tramitan en el Congreso Nacional brasileño. Se evidencia que los actuales proyectos de ley establecen amenazas fecundas en los principios orientadores, garantías, derechos y deberes para el uso de Internet en Brasil, camuflados en narrativas moralistas y de argumentos disonantes e interesados de la Ley $\mathrm{N}^{\circ}$ 12.965, llevando al entendimiento que hay eminentes posibilidades de ampliación del control y vigilancia de las informaciones que consumen y producen los internautas brasileños.
\end{abstract}

\begin{abstract}
The legalization and institutionalization of the surveillance of the digital information of the Brazilian citizens has been promoted by interests of Protestant political groups that argue ideas of faith and morality in draft laws that promote new forms of Internet control. Since 2015, different bills have threatened the right to privacy and freedom of expression in digital communications, guaranteed by Act No. 12.965, of April 23, 2014 (Civil Internet Framework). The present research analyzes the critical points of the projects of law of control of Internet promoted by evangelical politicians and justified in moral arguments of protection of children and adolescents. The present article establishes a theoretical dialogue with the postulates of Manuel Castells in an attempt to demystify the arguments in favor of the control and uses a qualitative investigation by means of a survey and analysis of content of the bills of laws that still are processed in the National Brazilian Congress. It is evident that the current draft legislation establishes fruitful threats to the guiding principles, guarantees, rights and duties for the use of the Internet in Brazil, camouflaged in moralistic narratives and dissonant and interested arguments of Law No. 12,965, leading to the understanding that there are eminent possibilities of expanding the control and monitoring of the information consumed and produced by Brazilian Internet users.
\end{abstract}

\section{Palabras clave | keywords}

Marco Civil Internet; Registro Nacional de Acceso a Internet; control de Internet en Brasil; análisis documental. Civil Internet Framework; National Registry of Internet Access; Internet control in Brazil; documentary analysis. 


\section{Introducción}

Poco más de un año antes de la solicitud de aceptación del proceso de destitución de la presidenta Dilma Rousseff, la Ley nº 12.965, del 23 de abril de 2014 -también conocida como «Marco Civil de Internet»-, fue sancionada. En ésta, temas como la neutralidad de Internet, el registro de datos de conexión y navegación, entre puntos que establecen derechos inalienables como la libertad de expresión, la privacidad, la intimidad de los usuarios y la inviolabilidad de las comunicaciones, están garantizadas, aunque estos principios se encuentran actualmente amenazados.

El proyecto de Ley 2390/2015, de la autoría del diputado federal Pastor Franklin y el 5096/2016, propuesto por el diputado federal Célio Silveira, amenazan la confidencialidad de datos de los internautas brasileños y esperan ser expuestos ante la Comisión de Ciencia y Tecnología, Comunicación e Informática (CCTCI) del Congreso brasileño por el diputado Misionero José Olimpio. Los proyectos de leyes referenciados ut supra ponen en cuestión el «Marco Civil de Internet» que es, hasta el momento, la única expresión legislativa que regula los derechos y deberes de ciudadanos en ambientes virtuales en Brasil.

Estos proyectos de normativa se tratan de iniciativas legislativas de la bancada evangélica de líderes políticos y religiosos en el Congreso Nacional, cuyos argumentos parten de la preservación de los derechos de niños y adolescentes; aunque esta justificación sucumbe a los reales intereses de mayor control de datos y de la navegabilidad del ciudadano brasileño por la propuesta de institucionalización de un «Registro Nacional de Acceso a Internet».

El presente artículo inicia discutiendo la dicotomía moralista y pragmática del control de la información en el paradigma de la sociedad de la información, mediante un análisis de los postulados de Manuel Castells, para posteriormente analizar, utilizando la técnica cualitativa de análisis de contenido de base interpretativa, la ley «Marco Civil de Internet», frente a las nuevas propuestas legislativas anteriormente referenciadas. Para el análisis de contenido se hace el levantamiento de datos con el mayor número de palabras proferidas en la Ley vigente, en los proyectos ut supra citados y el análisis de los principales puntos que el Comité Gestor de Internet explica contrarios al proyecto. Así la presente investigación intentará dilucidar si las propuestas legislativas 
significarían un mayor control de la vida digital de los ciudadanos brasileños por parte de las instituciones gubernamentales.

\section{Sociedad en red: Promesa y control}

En muchos países hemos visto evolucionar nuevas formas de control de la información en Internet afectando directamente la libertad de los ciudadanos que están sujetos a represión por parte de sus gobiernos (Murdoch \& Roberts, 2013). Los cambios políticos también evolucionan las formas de mantener el control de acceso a la información para los ciudadanos (Kerr, 2014). En la sociedad de la información, promesa y control parecen mezclarse con la misma potencia de la aglutinación de la producción y el consumo de las mercancías digitales. La promesa de libertad y democratización de la información se superpone a las reales políticas de control y acceso amplio de las informaciones por parte de imperios privados y Estados. Para Manuel Castells (2005) la tecnología es el nuevo modo de producción en nuestra actual sociedad, donde las informaciones pasan a ser incorporadas en el seno de una economía globalizada. Es significativo que el autor (op. cit) se centra en la productividad informacional como los motores apalancadores del crecimiento económico, ya que estos todavía organizan las sociedades alrededor de sus centenarias lógicas de apropiación de los procesos de trabajo y de acumulación de riquezas. Estos son los aspectos inmutables de las dinámicas en los que coexiste la dualidad capitalista de encanto y control, de riqueza y miseria. Conceptos dudosos contenidos en toda la trilogía de Castells: La Era de la información: Economía, sociedad y cultura. Puyosa (2015) también coincide en que están apareciendo nuevas formas de control del Estado en la sociedad de la información al investigar empíricamente el concepto que denomina «dilema del dictador» en el caso venezolano.

En la red encontramos la entrega de la «acción carismática» (Pierucci, 2003) por parte de los emprendedores que se arriesgan en el afán de tener su propio negocio en el ecosistema digital, la entrega del intelecto y talento de programadores, diseñadores, responsables de comunicación y marketing, administradores y todo el cuerpo colectivo de colaboradores y creadores de contenidos. Por el otro lado en las interacciones se encuentra el alma encantada de consumidores que confían y proporcionan su información a cambio de servicios digitales, convirtiéndose en usuarios de mercancías informativas que aún no han des- 
pertado al sesgo mediático detrás de una gratuidad en la prestación de servicios -aplicaciones, sitios web, ofertas, entre otras- que hasta ahora nunca habían sido necesarios para un bienestar social, pero que así se convierten por la masificación cíclica e interminable de la cultura consumista de comprar y descartar (Gorz, 2005). En ese juego de la sociedad en red también aparecen los gobernantes que, en busca de expandir sus nichos electorales, intentan utilizar la red para sus intereses personales por medio de políticas públicas, discursos reconfortantes o promesas en las campañas electorales.

Cleland \& Brodsky (2012) recuerdan en su obra que el periódico USA Today reveló que en 2004, 98\% de las donaciones a campañas federales efectuadas por funcionarios de Google fueron para los políticos del partido demócrata: "Eric Schmift apoyó a Barack Obama, participó activamente en su campaña y fue miembro del Consejo Consultivo del Presidente para Ciencia y Tecnología y de su Consejo de Transición sobre la Economía" (op. cit, p. 201). El sitio estadounidense de noticias Sputnick News, en abril de 2015, reveló que el fundador de WikiLeaks, Julian Assange, previó que Google podría desempeñar un papel crucial en la campaña presidencial de Hillary Clinton en 2016 pues, según el reportaje, el director de «Google Ideas», Jared Cohen, fue asesor de Clinton. En Brasil, distante al escenario de las aseveraciones de Assange, vemos actitudes semejantes por parte de los miembros líderes religiosos que asumen funciones políticas, como por ejemplo del grupo parlamentario de evangélicos del Congreso Nacional que desde 2015 proponen proyectos de Ley que endosan la relación de control del Estado para el ejercicio de derechos ciudadanos.

"La red no es garante de libertad, pero vuelve más difícil la opresión. La censura permite identificar y castigar al mensajero, pero no puede detener el mensaje", afirma Manuel Castells al periódico brasileño Zero Hora ${ }^{1}$. Castells (2005), al mencionar el eslogan de la campaña presidencial de Bill Clinton en 1992, constata que la globalización fue en ese gobierno la principal estrategia de la política económica estadounidense, mientras surgía una mayor desregulación y liberalización -doméstica e internacional- postulando así:

1 Entrevista ofrecida el 09/06/13. Disponible en: https://goo.gl/DbRsqK (25/06/17). 
A resposta é clara para o principal globalizador, o governo dos EUA: uma economia mais aberta e integrada é vantajosa para para as empresas estadunidenses. Isso se dá em razão da vantagem tecnológica, e da flexibilidade administrativa superior, de que gozam os EUA em comparação com o resto do mundo. Junto com a presença de longa data das multinacionais estadunidenses no mundo inteiro, e com a presença hegemônica dos EUA nas instituições internacionais de comércio e finanças, a globalização é primordial para aumentar a prosperidade econômica dos EUA, embora decerto não para todas as empresas, e não para todo o povo dos Estados Unidos. Esse interesse econômico estadunidense é algo de que Clinton e sua equipe econômica, especialmente Rubin, Summers e Tyson entendiam bem. Trabalharam com afinco para divulgar o evangelho liberal no mundo, aplicando a força econômica e política dos EUA quando necessárioº (Castells, 2005, p. 184).

En otras palabras, para Castells (op. cit), en ausencia de conflictos o choques externos -revoluciones políticas, guerras, desastres naturalesla transformación del capitalismo está dirigida por intentos para sostener el crecimiento económico, simplemente porque el propio sistema tiene una lógica expansionista. Las presiones competitivas internas de las economías nacionales recompensan a aquellas cuya productividad es más elevada. En consecuencia, Castells mantiene la idea de que si nosotros entendemos cómo cambia la sociedad contemporánea, tenemos que empezar analizando las estrategias implementadas por los agentes económicos apuntando al crecimiento de la eficiencia de éstas, cobrando en este contexto mayor sentido la famosa frase del sociólogo Daniel Bell "hacer mucho con poco" (citado por Stalder, 2006, p. 43).

2 "La respuesta es clara para el principal globalizador, el gobierno de EEUU: una economía más abierta e integrada es ventajosa para las empresas estadounidenses. Esto se da en razón de la ventaja tecnológica y de la flexibilidad administrativa superior de la que goza Estados Unidos en comparación con el resto del mundo. Junto con la presencia de larga data de las multinacionales estadounidenses en todo el mundo y con la presencia hegemónica de EEUU en las instituciones internacionales de comercio y finanzas, la globalización es primordial para aumentar la prosperidad económica de Estados Unidos, aunque ciertamente no para todas las empresas y no para todo el pueblo de los Estados Unidos. Este interés económico es algo que Clinton y su equipo económico, especialmente Rubin, Summers y Tyson entendían bien. Trabajaron con ahínco para divulgar el evangelio liberal en el mundo, aplicando la fuerza económica y política de EEUU cuando era necesario" (Traducción libre al español por el autor). 
Stalder (2006) comprende que la referencia a Bell no es baladí. En realidad, el trabajo de Bell desempeñó un papel importante para la crítica de Castells, en su teoría de «post-industrialismo», que dominó por mucho tiempo el análisis de los cambios económicos contemporáneos y favoreció su teoría. Entre los académicos que analizaron las transformaciones de la economía en el último cuarto del siglo XX, Daniel Bell sostuvo una posición prominente por un tiempo considerable. Desde la publicación de The Coming of Postindustrial Society, en 1973, la teoría de Bell ha ejercido una influencia profunda en el discurso sobre la economía en la era de la información. Su argumento básico fue ampliamente popularizado por futuristas como Alvin Toffler, particularmente en La Tercera Ola. Ambos autores detectaron un cambio histórico en los procesos de creación de mercancías para la provisión de servicios como actividad esencial de las economías avanzadas.

Esta transformación, según los autores citados en referencia, es más o menos igual en importancia a la Revolución Industrial, la cual desplazó el grueso de la actividad económica en extraer recursos de la naturaleza a través de la agricultura y la minería para la producción de mercancías en los mercados masivos. Stalder (2006, p. 43) nos recuerda que Bell declaró que "las sociedades industriales son mercancías que producen sociedades. La vida es un juego contra la naturaleza fabricada [...] Una sociedad postindustrial se basa en servicios. En consecuencia, ese es un juego entre personas".

Continuando con el análisis de Stalder (op. cit), Castells usó la teoría de post-industrialismo desde mediados de la década de los setenta como un realce para discutir con los argumentos contrarios. A pesar de su disgusto por el futurismo -tan característico de su análisis postindustrial- la perspectiva de Castells se superpone con los temas más importantes de Bell. En primer lugar, ambos sitúan primariamente cambios cruciales en el nivel de relaciones técnicas de producción (modos de desarrollo) y solo secundariamente al nivel de sus relaciones sociales (modos de producción). Esto les permite hablar al mismo tiempo sobre un profundo cambio en la forma en que se crean los principios económicos y sobre la continuidad de la forma en que los principios son apropiados. Esta distinción ha sido durante mucho tiempo una tesis central para el análisis de Castells, en especial en la trilogía de la «Era de la Información», más específicamente en el primer volumen La Sociedad 
en Red, donde el autor defiende una nueva forma de capitalismo que emergió en el último cuarto del siglo XX.

El propio argumento de Castells puede ser entendido como un intento de superar lo que él ve como fallas fundamentales en el enfoque, mientras las mantiene como un importante punto de referencia. La crítica de Castells al post-industrialismo culmina en su análisis sobre la reestructuración de una época: la crisis económica de la década de los setenta; y se desdobla en cuatro principales conceptos de la sociedad en red: i) la internacionalización de la economía; ii) el crecimiento de los mercados financieros globales; iii) las empresas de red; iv) y la individualización del trabajo.

La masificación de los nuevos servicios tecnológicos a través de la venta de espacios publicitarios en los enmarañados flujos de información revela las mismas viejas bases estructurales de acumulación y control. En el centro de este paradigma vemos el incontestable intento de mayor control de la información con intereses privados. Tal y como afirman Cleland y Brodsky (2012, p. 123), "el componente microeconómico es más importante. En él es en el que el tráfico o el comportamiento de los consumidores son analizados, permitiendo a Google prever dicho comportamiento, atraer a más usuarios y vender más anuncios".

La gratuidad en los servicios de las firmas que producen información y conocimiento a través de la innovación son ejemplos que se contextualizan en la (in)explicable ambivalencia. De hecho, en sus negocios se denota facilitar, agilizar y organizar nuestras vidas en las más variadas texturas generales de nuestras experiencias sociales, tal y como explica Silverstone (2005); y así lo hacen al considerar las necesidades creadas en la cíclica cultura de consumo (Bauman, 2008) donde el querer inmediato suplanta el deseo y la necesidad.

Parece que hoy, más que nunca, el escenario predicho por Toffler (2007) en La Tercera Ola, donde los consumidores se acercan más a la apropiación del modo de producción teniendo un estatus de productores-consumidores (prosumers) es más real cuando observamos el control de los insumos inmateriales, el control de la información. ¿Estaríamos ante una contradictoria economía del conocimiento en la constancia de la vigilancia? Para Gorz (2010, p. 12) "El valor del conocimiento en la economía capitalista es indefinible [...] Su precio no tiene un fundamento objetivo y es continuamente fluctuante", siguiendo esto la misma lógica del sistema capitalista: Control y promesa. 


\section{3. «Marco Civil de Internet» versus «Registro Nacional de Acceso a Internet»}

La Ley 12.965 sancionada el 23 de abril de 2014 por la presidenta Dilma Rousseff estableció derechos y deberes referentes al acceso a la información en Brasil. Se trata de una norma enmarcada en la Constitución brasileña que contribuye a mantener una Internet «libre y segura», aunque no ha estado exenta de ser un punto de continuos choques y debates acalorados en la esfera política de Brasil. Conocida como «Marco Civil de Internet», la ley apareció conjuntamente con otros 36 proyectos de similares propuestas temáticas e incluyó temas polémicos como la neutralidad y los derechos ciudadanos de acceso a la red. Este artículo destaca cuatro puntos recolectados en una de las colecciones de la serie Legislación, de la Edições Câmara publicado en 2014.

El primer punto de la propuesta de «Marco Civil de Internet» se refiere a la neutralidad relativa de las redes, que permite el control de tráfico por los operadores privados, buscando mantener mayor transparencia con el usuario, expresado en el capítulo tercero, artículo noveno:

II - agir com proporcionalidade, transparência e isonomia; [...] III informar previamente de modo transparente, claro e suficientemente descritivo aos seus usuários sobre as práticas de gerenciamento e mitigação de tráfego adotadas, inclusive as relacionadas à segurança da rede $^{3}$ (Marco Civil da Internet, 2014, p. 32).

Sobre el acopio de la información del usuario, el Marco garantiza en el plazo de hasta un año el almacenamiento de datos de conexión referente a la navegación (dirección IP utilizada, hora de conexión, entre otros) por parte de los proveedores de Internet. El objetivo, que al fondo pasaba a identificar al usuario, también lo protegería de eventuales delitos cibernéticos. Asimismo se justificaba en la protección a la intimidad personal del ciudadano, sancionando eventuales incumplimientos por parte de los proveedores:

Art. 10. A guarda e a disponibilização dos registros de conexão e de acesso a aplicações de internet de que trata esta Lei, bem como de dados pessoais

3 II - actuar con proporcionalidad, transparencia e isonomía; (...) III - informar previamente de manera transparente, clara y suficientemente descriptiva a sus usuarios sobre las prácticas de gestión y mitigación de tráfico adoptadas, incluidas las relacionadas con la seguridad de la red (traducción libre por el autor). 
e do conteúdo de comunicações privadas, devem atender à preservação da intimidade, da vida privada, da honra e da imagem das partes direta ou indiretamente envolvidas. [...] Art. 11. Em qualquer operação de coleta, armazenamento, guarda e tratamento de registros, de dados pessoais ou de comunicações por provedores de conexão e de aplicações de internet em que pelo menos um desses atos ocorra em território nacional, deverão ser obrigatoriamente respeitados a legislação brasileira e os direitos à privacidade, à proteção dos dados pessoais e ao sigilo das comunicações privadas e dos registros ${ }^{4}$ (Marco Civil da Internet, 2014, p. 33-34).

El tercer punto se refiere al registro de historial de navegación del usuario. De acuerdo con la propuesta original, los proveedores de Internet eran vetados de almacenar cualquier dato referente al historial de navegación del usuario -excepto cuando el poder judicial ordenara lo contrario-, siendo proporcionado a proveedores de contenido, como por ejemplo empresas de redes sociales, portales de noticias y entretenimiento o motores de búsqueda, que tenían algún servicio de hospedaje de la información:

Art. 14. Na provisão de conexão, onerosa ou gratuita, é vedado guardar os registros de acesso a aplicações de internet. [...] Art. 15. O provedor de aplicações de internet constituído na forma de pessoa jurídica e que exerça essa atividade de forma organizada, profissionalmente e com fins econômicos deverá manter os respectivos registros de acesso a aplicações de internet, sob sigilo, em ambiente controlado e de segurança, pelo prazo de 6 (seis) meses, nos termos do regulamento. [..] $§ 2^{\circ} \mathrm{A}$ autoridade policial ou administrativa ou o Ministério Público poderão requerer cautelarmente a qualquer provedor de aplicações de internet que os registros de acesso a aplicações de internet sejam guardados [... $]^{5}$ (Marco Civil da Internet, 2014, p. 36).

4 Art. 10. La custodia y la disponibilidad de los registros de conexión y de acceso a las aplicaciones de Internet de que trata esta Ley, así como de datos personales y del contenido de comunicaciones privadas, deben atender a la preservación de la intimidad, la vida privada, El honor y la imagen de las partes directa o indirectamente implicadas. [...] Art. 11. En cualquier operación de recolección, almacenamiento, custodia y tratamiento de registros, de datos personales o de comunicaciones por proveedores de conexión y de aplicaciones de Internet en que al menos uno de esos actos ocurra en territorio nacional, Se deben respetar obligatoriamente la legislación brasileña y los derechos a la privacidad, a la protección de los datos personales y al secreto de las comunicaciones privadas y de los registros (traducción libre por el autor).

5 Art. 14. En la provisión de conexión, onerosa o gratuita, es vedado guardar los registros de acceso a aplicaciones de internet. [...] Art. 15. El proveedor de aplicaciones de Internet constituido en forma de persona jurídica y que ejerza esa actividad de forma 
El cuarto punto polémico incluido en el Marco normaliza la responsabilidad de contenido en la red, expresados en la práctica de la "notificación y retirada del aire". Esta tipificación se refiere a materiales infractores protegidos por derechos de autor, difamatorios o calumniosos:

Art. 18. O provedor de conexão à internet não será responsabilizado civilmente por danos decorrentes de conteúdo gerado por terceiros. [...] Art. 19. Com o intuito de assegurar a liberdade de expressão e impedir a censura, o provedor de aplicações de internet somente poderá ser responsabilizado civilmente por danos decorrentes de conteúdo gerado por terceiros se, após ordem judicial específica, não tomar as providências para, no âmbito e nos limites técnicos do seu serviço e dentro do prazo assinalado, tornar indisponível o conteúdo apontado como infringente, ressalvadas as disposições legais em contrário. [...] Art. 20. Sempre que tiver informações de contato do usuário diretamente responsável pelo conteúdo a que se refere o art. 19, caberá ao provedor de aplicações de internet comunicar-lhe os motivos e informações relativos à indisponibilização de conteúdo, com informações que permitam o contraditório e a ampla defesa em juízo, salvo expressa previsão legal ou expressa determinação judicial fundamentada em contrário ${ }^{6}$ (Marco Civil da Internet, 2014, p. 37).

organizada, profesionalmente y con fines económicos deberá mantener los respectivos registros de acceso a aplicaciones de internet, bajo secreto, En un ambiente controlado y de seguridad, por el plazo de 6 (seis) meses, de conformidad con el Reglamento. [...] § 2o La autoridad policial o administrativa o el Ministerio Público podrán requerir cautelarmente a cualquier proveedor de aplicaciones de Internet que los registros de acceso a aplicaciones de Internet sean guardados [...] (traducción libre por el autor).

6 Art. 18. El proveedor de conexión a Internet no será responsabilizado civilmente por daños derivados del contenido generado por terceros. [...] Art. 19. Con el fin de asegurar la libertad de expresión e impedir la censura, el proveedor de aplicaciones de Internet sólo podrá ser responsabilizado civilmente por daños derivados del contenido generado por terceros si, después de una orden judicial específica, no Tomar las providencias para, en el marco y dentro de los límites técnicos de su servicio y dentro del plazo señalado, hacer no disponible el contenido señalado como infringente, salvo las disposiciones legales en contrario. [...] Art. 20. Siempre que tenga información de contacto del usuario directamente responsable del contenido a que se refiere el art. 19, corresponderá al proveedor de aplicaciones de internet comunicarle los motivos e informaciones relativos a la indisponibilidad del contenido, con informaciones que permitan el contradictorio y la amplia defensa en juicio, salvo expresa previsión legal o expresa determinación judicial fundamentada en contrario (traducción libre por el autor). 
En resumen, las políticas de neutralidad pasaron a legislar y proteger el tráfico de Internet permitiendo que el usuario sea informado de las políticas y condiciones del contrato. Con la vigente Ley de «Marco Civil de Internet» el usuario tendrá que dar autorización expresa para la recolección de sus hábitos de navegación y aunque se opte en no proporcionar la información, podrá ser impedido de utilizar los servicios si no acepta los términos establecidos por el sitio. Uno de los puntos de conflicto en relación al control de la información por empresas privadas y el gobierno se refiere a la obligatoriedad de almacenamiento de registros de conexión y navegación definido por un período determinado en la legislación, pero al mismo tiempo no presentando obligación de continuos almacenamientos en algunos casos explicitados por la Ley. Además, otro punto importante al derecho del usuario se refiere a que los derechos constitucionales se validan también para la realidad digital.

Sin embargo, dos años más tarde de la sanción del «Marco Civil de Internet», el Comité Gestor da Internet no Brasil (en lo sucesivo CGI.br) divulgó el 18 de octubre de 2016 una nota pública contraria a las propuestas legislativas que pretendían crear un «Registro Nacional de Acceso a Internet» (Cadastro Nacional de Acesso à Internet). Una de las atribuciones del CGI.br es la proposición de normas y procedimientos relativos a la regulación de las actividades en Internet. El órgano se mostró radicalmente contrario al proyecto de Ley liderado por el Frente Evangélico Parlamentario del Congreso Nacional, compuesto enteramente por diputados evangélicos, defensores de un mayor control del acceso a la información y vigilancia de la ciudadanía brasileña:

O COMITÊ GESTOR DA INTERNET NO BRASIL - CGI.br, após tomar conhecimento, no último dia 05 de outubro de 2016, da leitura do parecer de aprovação e do substitutivo apresentado aos Projetos de Lei $n^{\circ}$ s $2390 / 2015,3597 / 2015,5016 / 2016$ e 5096/2016, bem como do pedido de vista conjunto de parte dos parlamentares integrantes da Comissão de Ciência e Tecnologia, Comunicação e Informática/CCTCI, [...]VEM A PÚBLICO. Expressar grande preocupação com a proposta de criação do denominado "Cadastro Nacional de Acesso à Internet", o qual possui barreiras técnicas para a sua implantação, bem como negligencia o controle parental e soluções que contemplem todos os aspectos envolvidos para garantir uma Internet livre, aberta, democrática e que seja um ambiente seguro para as crianças e adolescentes.2. Argumentar que a pretensão de acautelar riscos inerentes à navegação na Internet 
- que podem e devem ser mitigados com a educação dos usuários e de seus responsáveis - não deve justificar a criação de controles inconsistentes e passíveis de serem burlados, e que atentariam contra princípios fundamentais consagrados pelo Marco Civil da Internet, criando nova gama de riscos aos cidadãos que se pretende proteger $^{7}$ (CGI.BR, 2016) . $^{8}$.

Los proyectos de Ley números 2390/2015, 3597/2015, 5016/2016 y 5096/2016, agrupados en la eventual Ley que alude al «Catastro Nacional de Acceso a Internet» y que aún se encuentran en trámite en el Congreso $\mathrm{Nacional}^{9}$, han sido fuertemente criticados por proponer modificaciones que amenazan los principales principios y derechos postulados en el «Marco Civil de Internet», explicado con anterioridad.

\section{Materiales y método}

Para la presente investigación se realizó un levantamiento de los principales proyectos de Ley que actualmente se encuentran en trámite en el Congreso Nacional brasileño y que denotan nuevas formas de control de Internet, para posteriormente realizar un análisis de contenido de base interpretativa asignado de forma cuantitativa a las llaves lexicales más frecuentes en estos documentos verbales (transcripciones) y documentos legislativos. Para ello se analizó el contenido de las principales expresiones verbales y no verbales de las leyes ya discutidas, comparando

7 EL COMITÉ GESTOR DE INTERNET EN BRASIL - CGI.br, tras tomar conocimiento, el último día 05 de octubre de 2016, de la lectura del dictamen de aprobación y del sustitutivo presentado a los Proyectos de Ley no 2390/2015, 3597/2015, 5016 / 2016 y 5096/2016, así como de la solicitud de vista conjunto de parte de los parlamentarios integrantes de la Comisión de Ciencia y Tecnología, Comunicación e Informática / CCTCI, [...] HACEN PÚBLICO. Expresar gran preocupación con la propuesta de creación del denominado "Catastro Nacional de Acceso a Internet", el cual tiene barreras técnicas para su implantación, así como descuida el control parental y soluciones que contemplan todos los aspectos involucrados para garantizar una Internet libre, abierta Democrática y que sea un ambiente seguro para los niños y adolescentes.2. Argumentar que la pretensión de salvaguardar riesgos inherentes a la navegación en Internet -que pueden y deben ser mitigados con la educación de los usuarios y de sus responsables- no debe justificar la creación de controles inconsistentes y pasibles de ser burlados, y que atentarian contra principios fundamentales consagrados Por Marco Civil de Internet, creando una nueva gama de riesgos para los ciudadanos que se pretende proteger (traducción libre por el autor).

8 Disponible en: https://goo.gl/rUYhwk (26/04/17).

9 Hasta la fecha de elaboración de este artículo el 01/08/17. 
el discurso presentado en estas unidades documentales con la visión de repudio de los documentos de posicionamiento oficial de la asociación que representa a la sociedad civil (CGI.br) contraria al proyecto de «Catastro Nacional de Acceso a Internet».

Las políticas públicas que promulgan el control de Internet, más que en los propios textos legislativos, evidencian poder por palabras escritas, verbalizadas y mediatizadas. En cuanto a la preponderante presencia de textos en los fenómenos sociales contemporáneos, sobre todo en las comunicaciones políticas gubernamentales, Boréus \& Bergström (2017) indican que los estudios de los medios y de la comunicación deben sobrellevar el análisis textual como metodología de investigación de los campos de las Ciencias Sociales y en especial, de la Comunicación. Con la finalidad de organizar los diferentes sesgos analíticos para debatir sobre el «poder», se analizarán los discursos escritos, verbalizados o publicados, tomando en consideración tanto los pronunciamientos políticos como las críticas mediáticas.

El análisis de contenido se utiliza además para la codificación en un prisma cuantitativo o cualitativo, con el fin de sistematizar, definir, categorizar o describir el significado de los textos. Al mismo tiempo, los objetos de investigación, aproximándose a los sesgos cuantitativos y cualitativos, incluyen en estos análisis la producción de contenido de los medios de comunicación tradicionales y digitales (Boréus \& Bergström, 2017).

$\mathrm{Al}$ escoger el análisis de contenido, el investigador puede hacer comparaciones basadas en cuantificaciones de diferentes elementos en textos, lo que puede ser necesario si el objetivo es revisar los cambios en el transcurso del tiempo de determinado contenido. La principal característica de este tipo de análisis es el entendimiento que al dimensionar la frecuencia de determinadas palabras se podrán percibir características exteriores al texto. El análisis de contenido puede concentrarse en los aspectos ideales e interpersonales de los textos, manteniéndose como método deductivo en la búsqueda de elementos hipotéticos y teóricos. Las principales críticas a este tipo de método se refieren a que no todo se puede generalizar en frecuencia, porque existe un mayor carácter analítico a lo que es más explícito que implícito, en especial en la técnica de la búsqueda de códigos, y en relación a cuestiones de validación y de interpretación del investigador al sobreponerse a la realidad de los contenidos, tal como explican O'Connell (1999), McMillan (2009), Krippendorff (2013), Schreier (2014) y Boréus \& Bergström (2017). 
Para esta investigación se utilizó la plataforma ATLAS.ti para hacer el levantamiento de la frecuencia de palabras claves (lexicones) más presentes en los textos escritos y/o verbales de los principales puntos entre los dos proyectos de Ley referenciados (PL 2390/2015 y 2390/2015), incluyendo una comparación con el «Marco Civil de Internet». Además, fueron cuantificadas las palabras clave presentes con mayor frecuencia en el «Marco Civil de Internet» (ver tabla 1) y la posición del Comité Gestor de Internet do Brasil (ver tabla 2).

\section{Resultados}

\section{Tabla 1. Análisis comparativo de frecuencia de palabras clave contenidas en el "Marco Civil de Internet" y en los proyectos de Ley 12.965/2014 y 2390/2015}

\begin{tabular}{|c|c|c|c|c|c|}
\hline Texto analizado & Autoria & \multicolumn{4}{|c|}{$\begin{array}{l}\text { Palabras con mayor frecuencia } \\
\text { textual (escrito y /o verbal) }\end{array}$} \\
\hline $\begin{array}{l}\text { (1) } \\
\text { Ley n. 12.965/2014 } \\
\text { MARCO CIVIL } \\
\text { DE } \\
\text { INTERNET }\end{array}$ & $\begin{array}{l}\text { Sancionada } \\
\text { por la presi- } \\
\text { denta Dilma } \\
\text { Rousseff }\end{array}$ & $\begin{array}{l}\text { "INTERNET" } \\
\text { Qtd: } 70\end{array}$ & $\begin{array}{l}\text { "APLI- } \\
\text { CAÇÕES" } \\
\text { Qtd: } 35 \\
\text { Palabra clave } \\
\text { no comisera- } \\
\text { da: "art" (43) }\end{array}$ & $\begin{array}{l}\text { "REGISTROS" } \\
\text { Qtd: } 35\end{array}$ & $\begin{array}{l}\text { "LEl" } \\
\text { Qtd: } 26 \\
\text { "ACESSO"; "DADOS"; } \\
\text { "CONEXÃO" } \\
\text { Qtd: } 23 \\
\text { "CONTEÚDO" (Qtd: } \\
\text { 20); "FORMA" } \\
\text { (Qtd: 19) }\end{array}$ \\
\hline $\begin{array}{l}\text { (2) } \\
\text { Proyecto de ley } \\
\text { 2390/2015 }\end{array}$ & $\begin{array}{l}\text { Pastor Franklin } \\
\text { (Partido Polí- } \\
\text { tico: PTdoB/ } \\
\text { MG) }\end{array}$ & $\begin{array}{l}\text { "INTER- } \\
\text { NET" } \\
\text { Qtd: } 27\end{array}$ & $\begin{array}{l}\text { "ACESSO" } \\
\text { Qtd: } 24\end{array}$ & $\begin{array}{l}\text { "CADASTRO" } \\
\text { Qtd: } 20\end{array}$ & $\begin{array}{l}\text { "PÚBLICO"; } \\
\text { "ADOLESCENTE"; } \\
\text { "CRIANÇA" } \\
\text { Qtd: } 11\end{array}$ \\
\hline $\begin{array}{l}\text { (3) } \\
\text { Proyecto de ley } \\
5016 / 2016\end{array}$ & $\begin{array}{l}\text { Célio Silveira } \\
\text { - (Partido } \\
\text { Político: PSDB/ } \\
\text { GO) }\end{array}$ & $\begin{array}{l}\text { "ACESSO" } \\
\text { Qtd: } 15\end{array}$ & $\begin{array}{l}\text { "INTERNET" } \\
\text { Qtd: } 12\end{array}$ & $\begin{array}{l}\text { "LEl" } \\
\text { Qtd: } 11\end{array}$ & $\begin{array}{l}\text { "APLICATIVOS"; } \\
\text { "ADOLESCENTE" } \\
\text { Qtd: } 9\end{array}$ \\
\hline $\begin{array}{l}\text { (4) Discurso } \\
\text { pronunciado en la } \\
\text { Sala das Sessões, en } \\
27 \text { de abril de } 2016 \\
\text { por Deputado Célio } \\
\text { Silveira }\end{array}$ & $\begin{array}{l}\text { Célio Silveira } \\
\text { - (Partido Po- } \\
\text { lítico: PSDB/ } \\
\text { GO) }\end{array}$ & $\begin{array}{l}\text { "VÍDEOS"; } \\
\text { "INTER- } \\
\text { NET" } \\
\text { Qtd: } 10\end{array}$ & $\begin{array}{l}\text { "LEl” } \\
\text { Qtd: } 07\end{array}$ & $\begin{array}{l}\text { "CLASSI- } \\
\text { FICAÇÃO"; } \\
\text { "VIOLÊNCIA" } \\
\text { Qtd: } 06\end{array}$ & $\begin{array}{l}\text { "INDICATIVA"; } \\
\text { "SíTIOS"; "CENAS"; } \\
\text { "SEXO"; } \\
\text { "ANOS" } \\
\text { Qtd: } 5\end{array}$ \\
\hline $\begin{array}{l}\text { (5) } \\
\text { Discurso pronun- } \\
\text { ciado en la Sessões, } \\
14 \text { de abril de } 2016 \\
\text { por Deputado Célio } \\
\text { Silveira }\end{array}$ & $\begin{array}{l}\text { Célio Silveira } \\
\text { - (Partido } \\
\text { Político: PSDB/ } \\
\text { GO) }\end{array}$ & $\begin{array}{l}\text { "ACESSO" } \\
\text { Qtd: } 15\end{array}$ & $\begin{array}{l}\text { "INTERNET"; } \\
\text { "LEI" } \\
\text { Qtd: } 12\end{array}$ & $\begin{array}{l}\text { "APLICATI- } \\
\text { VOS" } \\
\text { Qtd: } 9\end{array}$ & $\begin{array}{l}\text { "SITES" } \\
\text { "ADOLESCENTES"; } \\
\text { "CRIANÇAS" } \\
\text { Qtd: } 8\end{array}$ \\
\hline
\end{tabular}




\section{Tabla 2. Posición del Comité Gestor de Internet do Brasil contraria a los proyectos de Ley "Registro Nacional de Acceso a Internet»}

a. que la protección en línea de niños y adolescentes a través de la creación de un "Catastro Nacional de Acceso a Internet", el cual, en teoría, restringiría el acceso al contenido "inadecuado" clasificado de forma unilateral por los propios "proveedores de información, marginaliza el papel de los padres en ejercer control sobre qué tipo de contenido sus hijos o hijas deberían consumir, haciéndolos coadyuvantes en la conducción de esa parte crucial del proceso educativo;

b. que existen diversos programas de computadoras para el ejercicio de control parental en cuanto al contenido visitado por niños y adolescentes, lo que está garantizado como una opción de libre elección en cualquier terminal de acceso a Internet y que debe ser objeto de políticas públicas en los términos del artículo 29. de la Ley 12.965/2014 (Marco Civil de Internet). Tales tecnologías empoderan a los padres con la habilidad de controlar las informaciones consumidas por sus hijos e hijas, lo que está más de acuerdo con su papel de protagonistas de esa porción importante del proceso educativo y que forma parte de la libre planificación familiar asegurada por la Constitución Federal (Artículo 226, §7º);

c. que hay la necesidad de esfuerzos coordinados entre el Poder Público, la sociedad civil, la comunidad científica y tecnológica y los proveedores de conexión y aplicación para establecer de forma estructurada los parámetros para el tratamiento de la circulación de material inadecuado por Internet;

d. que la obligatoriedad de sistemas de control embarcados en los equipos vincularía a la industria de bienes de informática con la obligación de agregarles la suscitada nueva funcionalidad, lo que inevitablemente será repasado al consumidor final, generando, en último análisis, efectos colaterales para la universalización de la conectividad en el país, así como para la viabilidad de la actividad de las pequeñas y medianas empresas;

e. que en una red local (residencial o corporativa) puede haber decenas de usuarios, dado que la mayoría de las veces se comparte el mismo número IP público. Por lo tanto, el proveedor de conexión tendría que autentificar cada uno de los miles de accesos de los miles de puntos de su red, lo que no guarda relación con dicho intercambio de protocolo IP y, en particular, con la interfaz de los hardwares de los actuales aparatos routers de conexión a Internet. En este último caso, que incluye las redes inalámbricas, la autenticación se haría sólo en el acceso principal y sólo una vez, lo que imposibilita el control de cada usuario;

f. por último, que cualquier sistema de registro podría ser fácil de burlar. En primer lugar, porque podría haber una autenticación falsa, como la creación o la utilización de registros que no por sus verdaderos titulares. Segundo, porque hay varias herramientas de enmascaramiento de la conexión, lo que la haría no rastreable por los proveedores y, en última instancia, por el "Catastro Nacional de Acceso a Internet";

Fuente: CGI.BR (2016). 
Tabla 3. Análisis comparativo de frecuencia
de palabras clave contenidas en la tabla 2

\begin{tabular}{|c|c|c|c|c|c|}
\hline $\begin{array}{c}\text { Texto } \\
\text { analizado }\end{array}$ & Autoria & \multicolumn{4}{|c|}{ Palabras con mayor frecuencia textual (escrito) } \\
\hline $\begin{array}{l}\text { (6) } \\
\text { Nota oficial } \\
\text { da CGI.BR } \\
\text { (2016) }\end{array}$ & $\begin{array}{l}\text { Comité } \\
\text { Gestor da } \\
\text { Internet no } \\
\text { Brasil (CGl. } \\
\text { br) }\end{array}$ & $\begin{array}{l}\text { "INTERNET" } \\
\text { Qtd: } 6\end{array}$ & $\begin{array}{l}\text { "ACESSO" } \\
\text { Qtd: } 5\end{array}$ & $\begin{array}{l}\text { "CONTROLE"; } \\
\text { "CONEXÃO" } \\
\text { Qtd: } 4\end{array}$ & $\begin{array}{l}\text { "CONTEÚDO" } \\
\text { "PROVEDORES" } \\
\text { Qtd: } 3\end{array}$ \\
\hline
\end{tabular}

Además del objeto central de la investigación de las palabras "Internet" y "acceso", en la tabla 1 se muestran los diferentes argumentos utilizados en los textos escritos y hablados señalados en los textos (2), (3) y (4), como «adolescentes» y «niños«. Esto pone en meridiana evidencia los valores morales que contextualizan la posición de la bancada evangélica que discuten -y justifican- temas relativos a la familia en muchos de sus discursos para un asunto de eminente naturaleza técnica y que involucra cuestiones de control de la información del ciudadano. El objetivo, de acuerdo con los diputados evangélicos, es "prohibir el acceso de niños y adolescentes a sitios electrónicos con contenido inadecuado". Para ello, la propuesta establece la creación de un registro nacional de personas y sitios, con datos personales que deberán ser verificados para cada acceso. La solución señalada por el «Registro Nacional de Acceso a Internet» impondría la creación de una base de datos en la que quedarían registradas "entre otras informaciones, el nombre completo, dirección completa, número del documento oficial de identidad y número de registro en el Catastro de Personas Físicas (CPF) del Ministerio de Hacienda", además de una lista de sitios con contenido inapropiado para niños y adolescentes. También se da cuenta el carácter disonante entre los puntos de los textos (1) y el otro en la tabla 1, posibilitando que la impresión de que el nuevo proyecto se expanda más allá de la atención formalizada en Ley vigente «Marco Civil de Internet».

Por su parte, la tabla 2 muestra la posición del CGI.br en la nota oficial divulgando los puntos contrarios al nuevo proyecto de Ley, que va más allá de los argumentos que se extraen de la tabla 1, sobre todo en la mayor cantidad de palabras claves en los textos escritos y hablados. Estos hechos se suman y se observan con meridiana claridad en la 
cuantificación expresada en la tabla 3 como "controle", donde la raíz "control" expresa denotativamente que los argumentos de la Ley en cuestión pueden tener un interés subyacente.

\section{Conclusión}

La Comisión de Ciencia y Tecnología, Comunicación e Informática (CCTCI) de la Cámara de Diputados tiene en la agenda de votaciones el informe del proyecto de Ley (PL) № 2.390/2015, que consolidado al PL 5016/201, proponen la creación de un «Registro Nacional de Acceso de Internet» defendido por la bancada evangélica y por líderes religiosos políticos en el Congreso brasileño, quienes argumentan que la preocupación por la protección de niños y adolescentes viene ganando apoyo de parte de la población en Brasil.

Este artículo analizó cuantitativamente, por medio de un análisis de contenido de base interpretativa, la mayor incidencia (frecuencia) de palabras claves proferidas por discursos verbales y escritos sobre estos proyectos de Ley que amenazan puntos importantes del «Marco Civil de Internet». En comparación con los textos analizados del proyecto de Ley, se puede contrastar con los puntos críticos que el Comité Gestor de Internet do Brasil posicionó en 2016 en nota oficial. Se percibió que palabras como "adolescente", "niño" y "acceso" pueden confundirse con "control" de manera connotativa o incluso denotativa. Como futuras líneas de investigación, la presente investigación ha identificado que merece ser ampliado para un análisis cualitativo más aproximado en eventos futuros y concluye que el actual proyecto que tramita en el Poder Legislativo brasileño puede intensificar el uso de informaciones de los ciudadanos, datos de navegabilidad e histórico de las actividades mapeadas a través del registro, provocando una amenaza directa a los derechos de los usuarios.

\section{Referencias}

Boréus, K. \& Bergström, G. (2017). Content Analysis. En Bergström, Göran \& Boréus, Kristina (Eds.), Analyzing text and discourse: Eight approaches for the Social Sciences. London: Sage Publications.

Castells, M. (2005). A Sociedade em Rede ( $8^{\underline{a}}$ ed). São Bernardo do Campo: Paz e Terra. (2008). O Poder da identidade (6 $6^{\mathbf{a}}$ ed). São Paulo: Paz e Terra.

Cleland, S. \& Brodsky, I. (2012). Busque e destrua: por que você não pode confiar no Google Inc. São Paulo: Matrix. 
Dovring, K. (2009). Quantitative Semantics in 18th Century Sweden. En K. Krippendorff \& M.A. Bock (Eds.), The Content Analysis Reader. London: Sage.

Gorz, A. (2005). O imaterial: conhecimento, valor e capital. São Paulo: Annablume.

Kerr, O. S. (2014). The Fourth Amendment and the Global Internet. GWU Law School Public Law Research Paper No. 2014-30; GWU Legal Studies Research Paper No. 2014-30. Disponible en SSRN: https://ssrn.com/abstract $=2428042$

Marco Civil da Internet (2014). Câmara dos Deputados, Brasil. Brasilia: Edições Câmara. McMillan, S.J. (2009). The Challegen of applying Content Analysis to the WorldWide Web. En K. Krippendorff \& M.A. Bock (Eds.), The Content Analysis Reader. London: Sage.

Murdoch, S. J. \& Roberts, H. (2013). Internet Censorship and Control. IEEE Internet Computing, 17(3), 6-9. http://dx.doi.org/10.1109/mic.2013.51

O'Connell, M. (1999). Is Irish public opinion towards crime distorted by media bias? European Journal of Communication, 14(2), 191-212.

Pierucci, A. (2003). O desencantamento do mundo: todos os passos do conceito em Max Weber. São Paulo: Editora 34.

Puyosa, I. (2015). Control politico de internet en el contexto de un régimen híbrido. Venezuela 2007-2015. Teknokultura, 12(3), 501-526. http://dx.doi. org/10.5209/rev_tk.2015.v12.n3.50392.

Schreier, M. (2014). Qualitative Content Analysis? En U. Flick (Eds.), The Sage Handbook of Qualitative Data Analysis. London: Sage.

Silverstone, R. (2005). Por que estudar a mídia? (2ª ed). São Paulo: Edições Loyola. 